\title{
Obstructive sleep apnea-induced multi-organ dysfunction after elective coronary artery bypass surgery in coronary heart disease patients
}

\author{
Jiayang Wang ${ }^{1,2}$, Xinxin Wang ${ }^{3}$, Wenyuan Yu ${ }^{1}$, Kui Zhang ${ }^{1}$, Yongxiang Wei ${ }^{4}$ \\ ${ }^{1}$ Department of Cardiac Surgery, Beijing An Zhen Hospital, Capital Medical University, Beijing, China; ${ }^{2}$ Center for Cardiac Intensive Care, Beijing \\ An Zhen Hospital, Capital Medical University, Beijing, China; ${ }^{3}$ Department of General Surgery, Chinese PLA general hospital, Beijing, China; \\ ${ }^{4}$ Department of Otolaryngology Head \& Neck Surgery, Beijing Anzhen Hospital, Capital Medical University, Beijing, China \\ Contributions: (I) Conception and design: J Wang, Y Wei; (II) Administrative support: None; (III) Provision of study materials or patients: None; (IV) \\ Collection and assembly of data: W Yu, X Wang; (V) Data analysis and interpretation: K Zhang; (VI) Manuscript writing: All authors; (VII) Final \\ approval of manuscript: All authors. \\ Correspondence to: Dr. Yongxiang Wei. Department of Otolaryngology Head \& Neck Surgery, Beijing Anzhen Hospital, Capital Medical University, \\ 2 Anzhen Road, Chaoyang District, Beijing 100029, China. Email: weiyongxiang@vip.sina.com; Dr. Jiayang Wang. Department of Cardiac Surgery, \\ Beijing An Zhen Hospital Capital Medical University, Beijing, China. Email: athlandwang@hotmail.com.
}

Background: The aim of this study was to explore the underlying impact of obstructive sleep apnea (OSA) on postoperative parameters of multi-organ function among coronary heart disease (CHD) patients following elective coronary artery bypass grafting (CABG).

Methods: Electronic literature databases were searched manually and automatically for relevant English articles. All of the included articles focused on a comparison of the incidence of postoperative parameters of multi-organ function in CHD patients undergoing elective CABG with and without OSA. Studies were excluded if they met any one of the following criteria: (I) duplicate publication; (II) ongoing or unpublished studies; (III) only published as abstracts or conference proceedings; and (IV) less than 30 patients in the patient cohort.

Results: A total of 13 articles met our inclusion criteria. The current study demonstrated OSA significantly increased the incidence of major adverse cardiac and cerebrovascular events (MACCEs) in CHD patients undergoing elective CABG compared with the controls [odds risk (OR), 1.97; 95\% CI, 1.50 to 2.59 , $\mathrm{P}<0.0001]$. In addition, OSA was associated with an increased risk of new revascularization in CHD patients undergoing elective CABG (OR, 9.47; 95\% CI, 2.69 to 33.33, $\mathrm{P}<0.0001)$. Moreover, reintubation and tracheostomy in the OSA group was increased $243 \%$ (OR, 3.43; 95\% CI, 1.35 to $8.71 ; \mathrm{P}=0.009$ ) and $372 \%$ (OR, 4.72; 95\% CI, 1.23 to 18.13; $\mathrm{P}=0.024$ ), respectively, compared with the control group. Besides, we also confirmed OSA significantly increased the acute kidney injury (AKI) incidence by 124\% (OR, 2.24; $95 \%$ CI, 1.07 to $4.72 ; \mathrm{P}<0.0001)$.

Conclusions: OSA may contribute to postoperative multi-organ dysfunction among CHD patients undergoing elective CABG by increasing the incidence of MACCEs, especially new revascularization, as well as respiratory, and renal complications.

Keywords: Obstructive sleep apnea (OSA); coronary heart disease (CHD); elective coronary artery bypass grafting (CABG); postoperative multi-organ dysfunction

Submitted May 26, 2020. Accepted for publication Aug 28, 2020.

doi: $10.21037 /$ jtd-20-2037

View this article at: http://dx.doi.org/10.21037/jtd-20-2037

(C) Journal of Thoracic Disease. All rights reserved. 


\section{Introduction}

Obstructive sleep apnea (OSA), a syndrome caused by repeated narrowing of the throat during sleep, is a common breathing-associated sleep disorder with recent estimates of prevalence in the general adult population ranging from $9 \%$ to $38 \%(1,2)$. In coronary heart disease (CHD) patients undergoing elective coronary artery bypass grafting (CABG), the incidence of mild OSA [ $5 \leq$ apnea-hypopnea index $(\mathrm{AHI})<15]$ and moderate to severe OSA $(\mathrm{AHI} \geq 15)$ is $74 \%$ and $48 \%$, respectively (3). Despite the high prevalence of OSA among CHD patients following CABG, the impact of OSA in the postoperative multi-organ function is not well established. Most of the evidence is focused on arrhythmia recurrence after cardiac surgery $(4,5)$, the effects of OSA on respiratory as well as renal parameters still need to be investigated further. In addition, as for the cardio-cerebrovascular complications, recent studies are based on patients diagnosed with different cardiac diseases undergoing various types of elective cardiac surgeries (6-8). Data on CHD patients developing postoperative complications after CABG are sparse. So far, no meta-analyses have focused on documenting a direct correlation between CHD patients following elective CABG with OSA and a higher risk of adverse cardiac and cerebral vascular events.

This current meta-analysis was initiated to fill the above knowledge gaps. We particularly focused on CHD patients with or without OSA. The aim of this study was to explore the underlying impact of OSA on postoperative parameters of multi-organ function, including cardiac and cerebral vascular, respiratory as well as renal postoperative complications, among CHD patients following elective CABG. Recognition of the association and determinants of OSA on postoperative multiple organ dysfunction should lead to strategies to improve the prognosis of CHD patients undergoing elective CABG.

\section{Methods}

\section{Literature search and selection criteria}

This systematic review and meta-analysis were conducted and reported in adherence to the Preferred Reporting Items for Systematic Reviews and Meta-Analyses (PRISMA) reporting checklist (9) (available at http://dx.doi. org/10.21037/jtd-20-2037). Electronic literature databases, including PubMed, ISI Web of Science, Directory of Open Access Journals, and the Cochrane Library electronic databases, were searched manually and automatically for relevant English articles. All of the selected articles focused on a comparison of the incidence of primary and secondary outcomes in CHD patients undergoing elective CABG with and without OSA.

As for further full-text assessment. The references of the retrieved articles were also reviewed to identify additional eligible studies. The study selection program was shown in Figure 1. Studies meeting the following criteria were included:

The following search terms were used: ("sleep apnea" [All Fields] OR "sleep apnea" [All Fields]) AND ("CABG" [All Fields] OR "coronary artery bypass grafting” [All Fields]) AND ("major adverse cardiac and cerebrovascular events" [All Fields] OR “cardiovascular outcomes" [All Fields] OR "major cardiovascular and cerebrovascular adverse events" [All Fields]).

The last search was conducted on July 1, 2019. Two investigators (J.W. and W.Y.) performed the initial search separately, deleted duplicate records, screened titles and abstracts for relevance, and identified relevant article:

(I) Patients: subjects were CHD patients following elective CABG.

(II) Intervention: elective CABG.

(III) Comparison: CHD patients undergoing elective CABG with preoperative OSA vs. CHD patients undergoing elective CABG without preoperative OSA.

(IV) Outcomes: multi-organ parameters, including cardiac, respiratory and renal parameters; in-hospital death; infection; intensive care unit (ICU)-readmission; length of ICU stay; length of hospital stay.

(V) Study design: observational studies (prospective or retrospective cohort studies) were included in this meta-analysis.

Studies were excluded if they met any one of the following criteria: (I) duplicate publication; (II) ongoing or unpublished studies; (III) only published as abstracts or conference proceedings; and (IV) less than 30 patients in the patient cohort.

\section{Data extraction and quality assessment}

Two reviewers (J.W. and W.Y.) independently extracted the following information from the eligible studies: first author, year of publication, country, study design, patient characteristics, number of patients enrolled, intervention, and outcome data. When the same patients were reported in several publications, only the largest study was retained to avoid duplication of information. The current study used the Downs and Black score system to evaluate the quality 


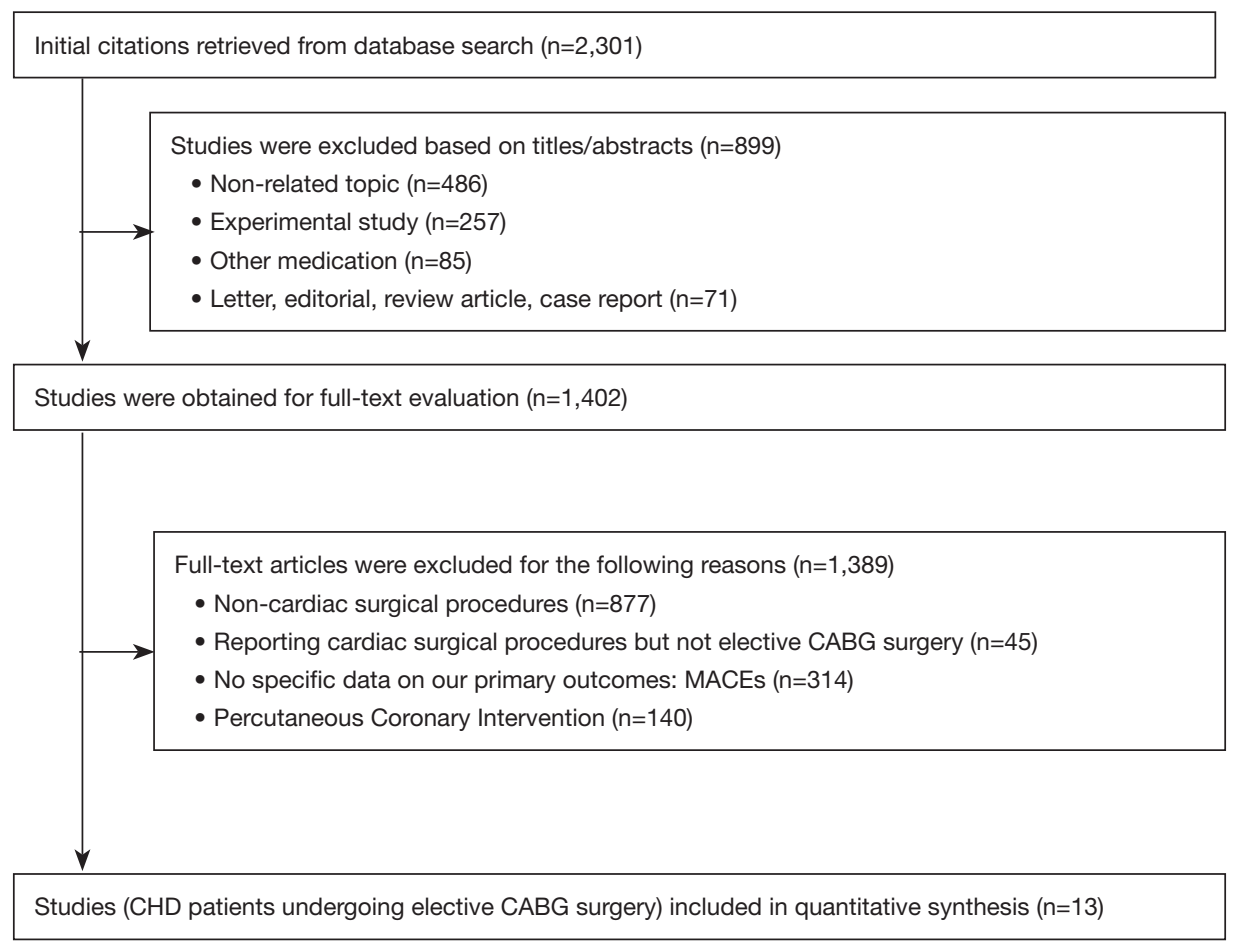

Figure 1 Flow diagram of study selection.

of each study. The quality of studies with a score $\geq 20$ was considered good and a score $<20$ was considered poor.

\section{Study end points and OSA definition}

The AHI was defined as the average number of episodes of apneas and hypopneas per hour of sleep. OSA must be diagnosed by polysomnography (PSG), which was defined in events per hour as AHI $\geq 5$ or screened as "high-risk OSA" by screening questionnaires.

Diagnosis of OSA and pre-/postoperative continuous positive airway pressure (CPAP) use in patients with OSA were shown in Table 1. Endpoints were as follows: (I) A composite end point of major adverse cardiac and cerebrovascular events (MACCEs) up to 30 days after surgery, including: cardiac death, myocardial infarction (MI), myocardial injury, nonfatal cardiac arrest, new revascularization (PCI or CABG), deep venous thrombosis, newly documented postoperative atrial fibrillation (POAF), postoperative cerebral vascular accident (CVA) or transient ischemic attack (TIA), and congestive heart failure. MACCEs and variables of major morbidity defined as in the Society of Thoracic Surgeons (STS) national database (10). (II) Respiratory complications were respiratory failure or major pulmonary complications. Postoperative respiratory failure was defined as the need for prolonged mechanical ventilation of $>24 \mathrm{~h}$ or the development of airway complications, including the need of reintubation or tracheostomy. Acute respiratory distress syndrome (ARDS), pneumonia, or pulmonary embolism represented major pulmonary complications. Renal complications included postoperative acute kidney injury (AKI) according to the KDIGO (Kidney Disease: Improving global outcomes) criteria.

\section{Statistical analysis}

Differences are expressed as odds risk (OR) or standardized mean difference (SMD) with $95 \%$ CI. Study heterogeneity was tested using the $\mathrm{I}^{2}$ statistic. Heterogeneity was considered to be low in studies with an $\mathrm{I}^{2}$ between $25 \%$ and $50 \%$, moderate in studies with an $\mathrm{I}^{2}$ between $50 \%$ and $75 \%$, and high in studies with an $\mathrm{I}^{2}$ exceeding $75 \%$. $\mathrm{I}^{2}$ exceeding $50 \%$ represents significant heterogeneity. A fixed-effects model was used when study heterogeneity was not significant; a random-effects model was used when study heterogeneity was significant. Funnel plots and the Egger linear regression test were used to estimate publication bias. Meta-regression analysis was performed to examine whether the pooled outcomes could be modified by the confounding factor. A Q 
Table 1 Diagnosis of OSA and pre-/postoperative CPAP use in patients with OSA

\begin{tabular}{|c|c|c|c|c|}
\hline Reference & $\begin{array}{l}\text { Screening and/or } \\
\text { diagnostic tool }\end{array}$ & $\begin{array}{l}\text { Patients on preoperative } \\
\text { CPAP use }(n)\end{array}$ & $\begin{array}{c}\text { Patients on postoperative } \\
\text { CPAP }\end{array}$ & $\begin{array}{c}\text { when was screened for } \\
\text { OSA }\end{array}$ \\
\hline Mooe et al. & PSG & None & None & Before surgery \\
\hline Bhama et al. & PSG & None & None & Before surgery \\
\hline Amra et al. & Questionnaire & NR & NR & Before surgery \\
\hline Oosten et al. & PSG & $63 \%$ & None & Before surgery \\
\hline Wong et al. & PSG & $44 \%$ & None & Before surgery \\
\hline Patel et al. & Questionnaires & $100 \%$ & $100 \%$ & Before surgery \\
\hline Tafelmeier et al. & PSG & NR & NR & Before surgery \\
\hline
\end{tabular}

OSA, obstructive sleep apnea; CPAP, continuous positive airway pressure; NR, not report; PSG, polysomnography.

model statistic was used in the meta-regression. All $\mathrm{P}$ values are two-sided. Statistical analyses were conducted by using Stata 15.0 software (StataCorp LP, College Station, TX).

\section{Results}

\section{Study selection, characteristics and quality assessment}

The selection process is illustrated in Figure 1. A total of 13 articles met our inclusion criteria $(3,11-22)$. The main characteristics of the included studies are described in Table 2. Total primary and secondary pooled outcomes in patients with and without OSA were demonstrated in Table 3. Of the 13 reports, three contained CHD patients following elective CABG + valvular surgery $(3,12,19)$. Meta-regression analysis was performed to examine whether the above pooled outcomes could be modified by the valvular surgery. The results were shown in Table 3. In addition, Downs and Black Score analysis revealed that all of the 13 articles had good quality (score $\geq 20$, Table 2).

\section{The impact of OSA on postoperative cardiac and cerebrovascular parameters in CHD patients following elective CABG (primary outcomes, Figure 2, Table 3)}

The current study demonstrated OSA significantly increased the incidence of MACCEs in CHD patients undergoing elective CABG compared with the controls (OR, 1.97; $95 \%$ CI, 1.50 to 2.59, $\mathrm{P}<0.0001$; Figure $2 A$ and Table 3) without significant heterogeneity $\left(\mathrm{I}^{2}=9.3 \%\right)$ and publication bias $(\mathrm{P}=876)$. The funnel plot also showed low publication bias (Figure 3). No significant effects were detected in meta-regression analysis with respect to the examined covariate $(\mathrm{P}=0.665$, Table 3); namely, the valvular surgery. In addition, OSA was associated with an increased risk of new revascularization, including $C A B G$ and percutaneous coronary intervention (PCI), in CHD patients undergoing elective CABG (OR, 9.47; 95\% CI, 2.69 to 33.33, $\mathrm{P}<0.0001$; Figure $2 D$ and Table 3) without significant heterogeneity $\left(\mathrm{I}^{2}=0.0 \%\right)$. Since all the included patients in this endpoint underwent CABG alone, the confounding factor had no effects on the pooled outcome. Furthermore, the current meta-analysis confirmed OSA did not affect the incidence of postoperative MI and CVA or TIA (Figure 2B,C and Table 3).

\section{The impact of OSA on postoperative respiratory and renal parameters in CHD patients following elective CABG (secondary outcomes, Figures 4,5, Table 3)}

The current study demonstrated that reintubation in the 
Table 2 Patient characteristics and down and black scores

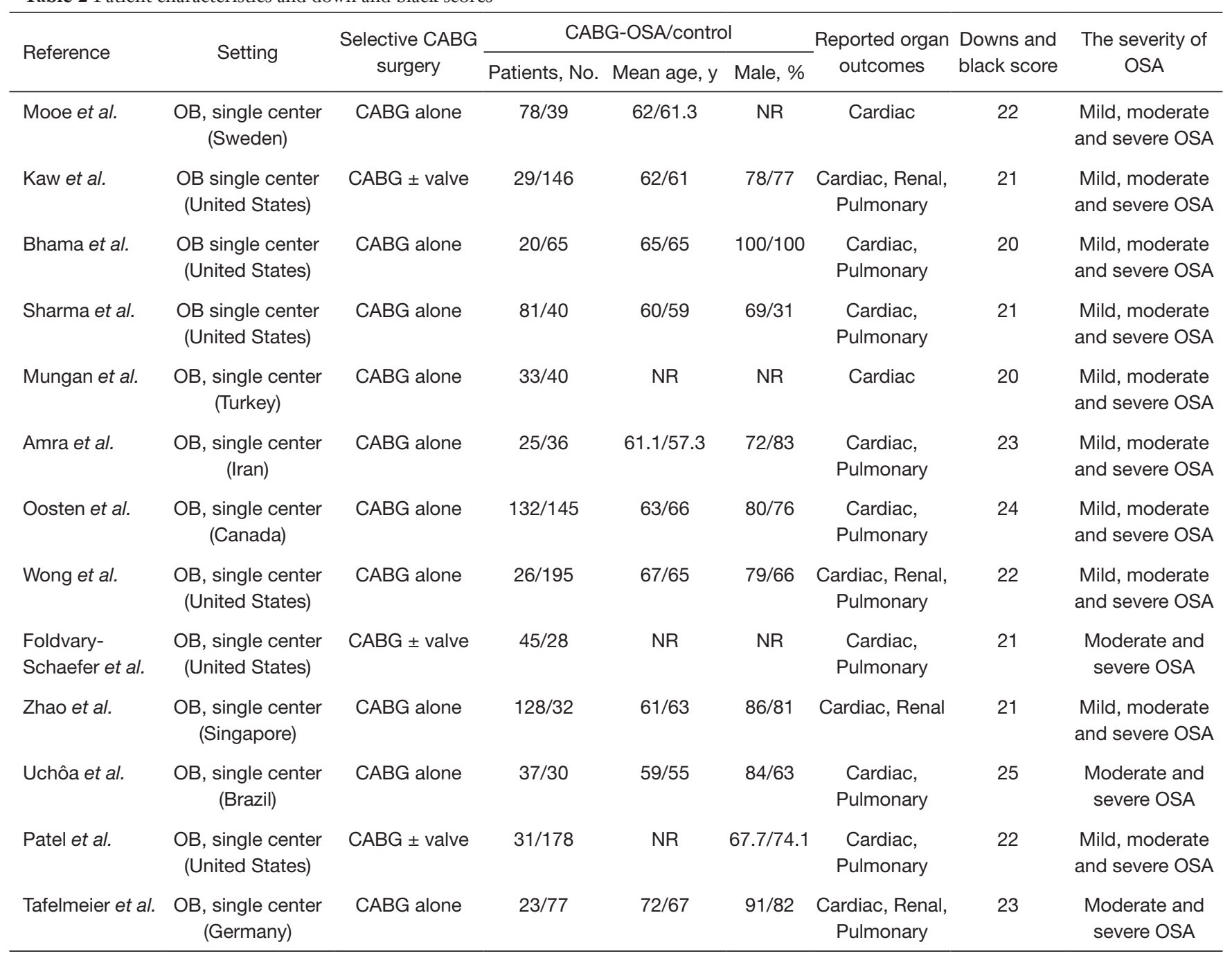

CABG, Coronary Artery Bypass Grafting; OSA, obstructive sleep apnea; OB, observational study

OSA group was increased by $243 \%$ compared with the control group (OR, 3.43; 95\% CI, 1.35 to $8.71 ; \mathrm{P}=0.009$; Figure $4 B$, Table 3) but with significant heterogeneity $\left(\mathrm{I}^{2}=50.0 \%\right)$. Meanwhile, patients with OSA group showed a $372 \%$ increase in tracheostomy compared with the control group (OR, 4.72; 95\% CI, 1.23 to 18.13 ; $\mathrm{P}=0.024$; Figure 4C, Table 3) without significant study heterogeneity $\left(\mathrm{I}^{2}=0.0 \%\right)$. Furthermore, we found OSA did not affect the incidence of postoperative major pulmonary complications and pulmonary edema (Figure $4 A, D$ and Table 3). In metaregression analysis, we found the examined covariate, namely, the valvular surgery, had no significant effect on the above pooled outcomes.

We also confirmed OSA significantly increased the
AKI incidence by $124 \%$ (OR, 2.24; $95 \%$ CI, 1.07 to 4.72; $\mathrm{P}<0.0001$; Figure 5 and Table 3) without significant study heterogeneity $\left(\mathrm{I}^{2}=0.0 \%\right)$. In meta-regression analysis, we found the examined covariate, namely, the valvular surgery, had no significant effect on the above pooled outcomes.

\section{The impact of OSA on postoperative exploratory parameters in CHD patients undergoing elective $C A B G$ (exploratory outcome, Table 3)}

Our results demonstrated that OSA increased medical resource utilization including length of postoperative hospital stay (SMD, 0.30; 95\% CI, 0.12 to 0.47 ; $\mathrm{P}<0.0001$; Table 3) and ICU stay (SMD, 0.30; 95\% CI, 0.16 to 0.44; 


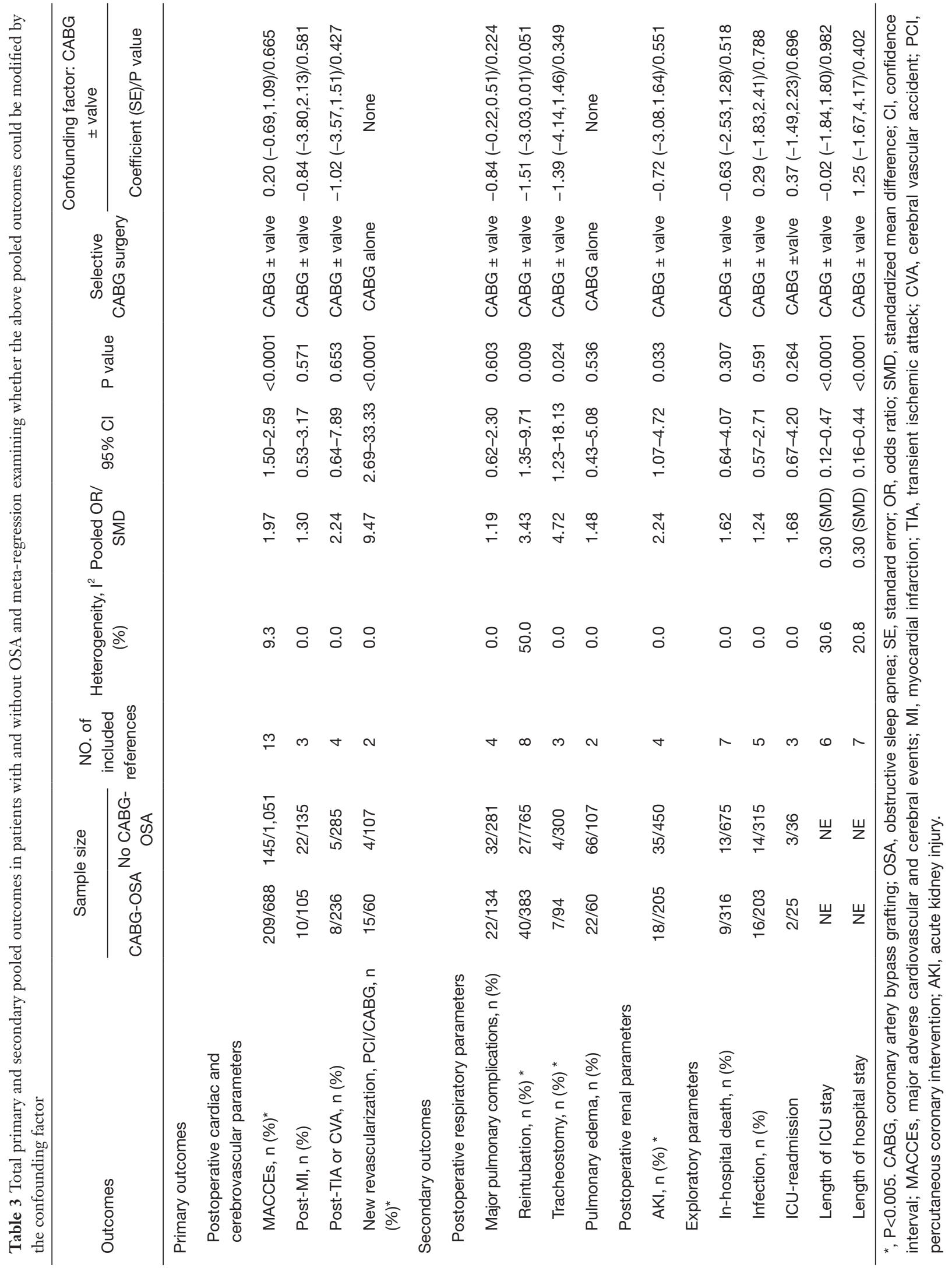




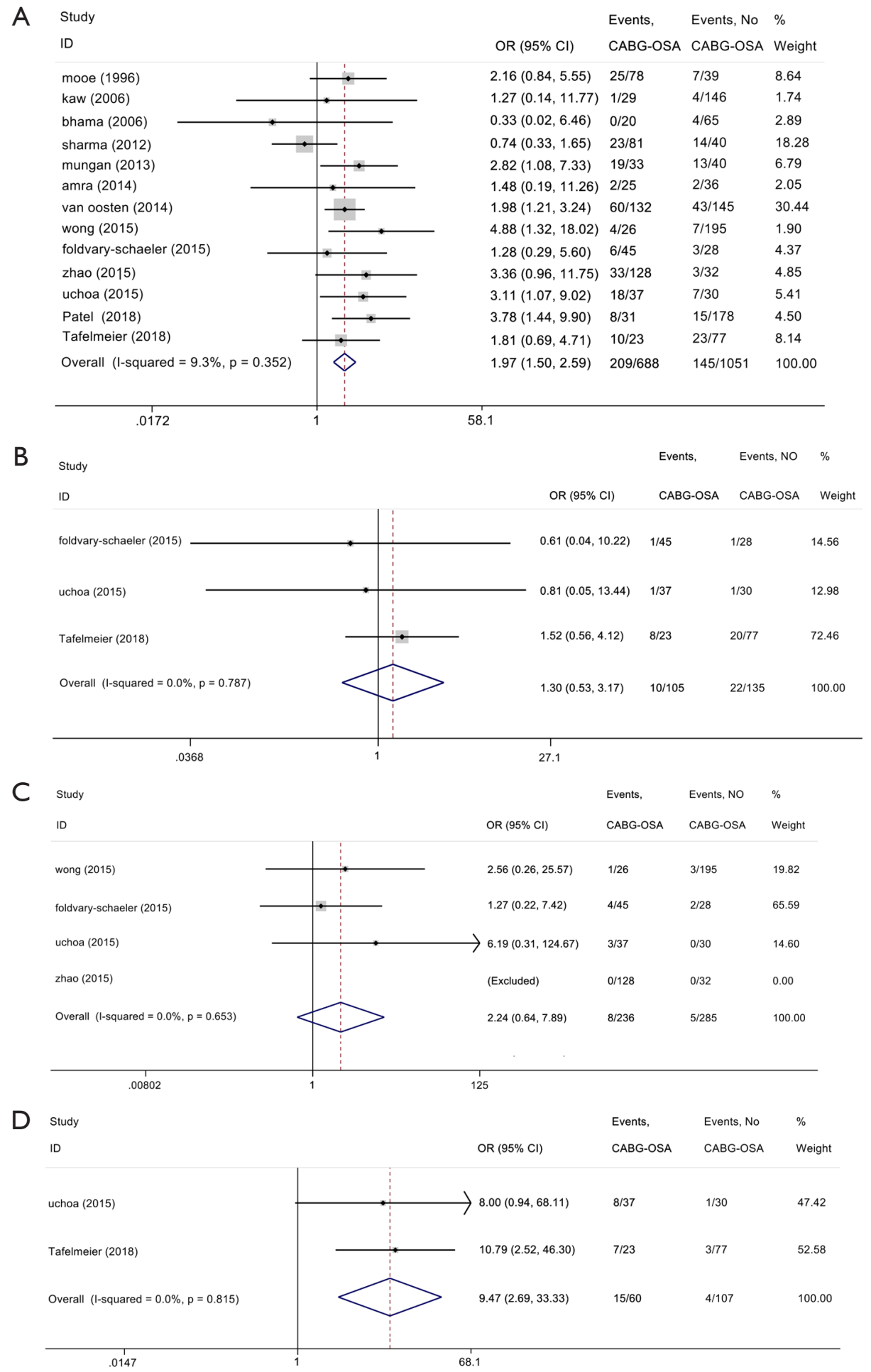

Figure 2 The impact of OSA on postoperative cardiac and cerebrovascular parameters in CHD patients following elective CABG. Cardiac and cerebrovascular parameters: (A) MACCEs; (B) MI; (C) CVA or TIA; (D) New revascularization, including CABG and PCI. OSA, obstructive sleep apnea; CHD, coronary heart disease; CABG, coronary artery bypass grafting; MACCEs, major adverse cardiac and cerebrovascular events; MI, myocardial infarction; CVA, cerebral vascular accident; TIA, transient ischemic attack; PCI, percutaneous coronary intervention; OR, odds risk; CI, confidence interval. 


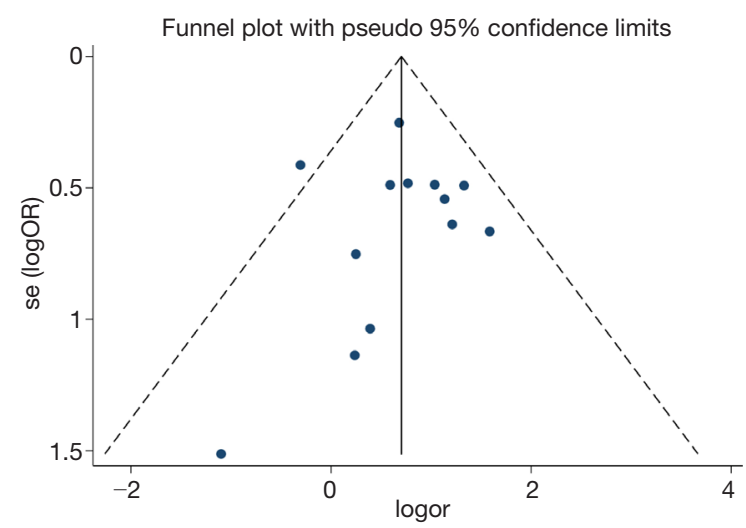

Figure 3 The funnel plot shows low publication bias.

$\mathrm{P}<0.0001$; Table 3) without significant study heterogeneity. On the contrary, OSA did not affect the incidence of postoperative in-hospital death, infection and ICUreadmission (Table 3). In meta-regression analysis, we found the examined covariate, namely, the valvular surgery, had no significant effect on the above pooled outcomes.

\section{Meta-regression Analysis of the Potential Modifiers}

Meta-regression analysis demonstrated the above pooled outcomes could not be modified by the valvular surgery (Table 3). Furthermore, the results also shown the above pooled outcomes were not affected by the following modifiers, including confirmation of OSA (PSG or not); quality of study; loss of patients due to follow-up and OSA with CPAP treatment (Table 4).

\section{Discussion}

To the best of our knowledge, this is the first meta-analysis to comprehensively examine the association between OSA and postoperative multi-organ dysfunction in CHD patients undergoing elective CABG. Our key findings are: (I) compared with the control group, OSA significantly increased the incidence of MACCEs in CHD patients undergoing elective CABG. In addition, OSA was associated with an increased risk of new revascularization, including CABG and PCI, in CHD patients undergoing elective CABG; (II) reintubation in the OSA group was increased compared with the control group. Meanwhile, patients with OSA group showed an increase in tracheostomy compared with the control group; (III) OSA significantly increased the AKI incidence compared with the control group; (IV) we also confirmed the association between OSA and increased medical resource utilization.

In the past 10 years, only one meta-analysis had investigated the effects of preoperative OSA on postoperative MACCEs among patients following cardiac surgery (6). However, the heterogeneity of the results cannot be ignored $\left(\mathrm{I}^{2}=64 \%\right)$. Previous meta-analysis focused on patients with varying cardiovascular diseases, including CHD, valvular heart disease and aortic disease, which might account for the source of the huge heterogeneity and led to inaccurate pooled results. In this current meta-analysis, we reduced the heterogeneity by including only $\mathrm{CHD}$ patients alone. For CHD patients undergoing CABG + the valvular surgery, meta-regression showed no significant effect on our pooled outcomes. In addition, there is another limitation in the previous meta-analysis. Insufficiency of the pooled postoperative endpoints other than MACCEs had impeded meaningful conclusions in cardiac surgical patients. The current research systematically examined the association between OSA and postoperative multi-organ dysfunction, including the parameters of cardiac and cerebral vascular, respiratory and renal function, in CHD patients undergoing elective CABG. The findings of this study might shed new light on optimizing the preoperative risk assessment for postoperative multi-organ dysfunction as well as the peri- and postoperative management of CHD patients undergoing elective CABG.

Previous studies investigating the effects of OSA on postoperative cardiac events among CHD patients following CABG show controversial results. For instance, $\mathrm{Ucho}^{\wedge} \mathrm{a}$ and colleagues (14) did not find a significant increase in MACCEs (including POAF) among CHD patients undergoing CABG with OSA in the short-term follow-up. On the contrary, Tafelmeier and colleagues (13) demonstrated sleep-disordered breathing, particularly OSA, is associated with adverse cardiac events after CABG, independent of known confounders. The findings of our study are in accordance with previous studies that found OSA significantly increased the incidence of MACCEs in CHD patients undergoing elective CABG. Notably, the current meta-analysis firstly confirmed OSA was associated with an increased risk of new revascularization. The discovery may have far more profound implications for peri- and postoperative management of CABG. Since previous studies showed that new or repeated revascularization in patients with triple-vessel disease independently increased the risk for ICU-associated adverse outcomes and postoperative hospital and ICU 
A

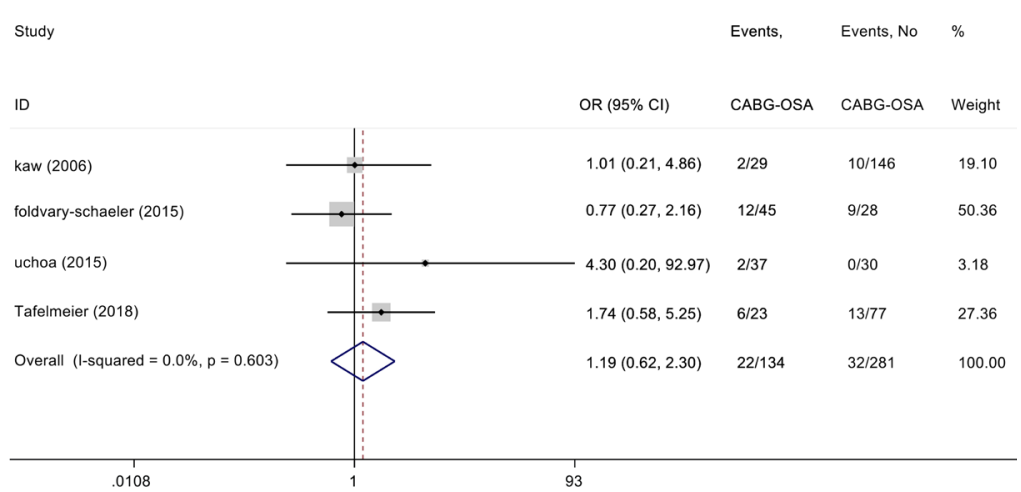

B

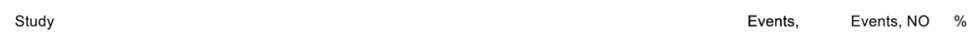

ID

OR $(95 \% \mathrm{Cl})$

CABG-OSA CABG-OSA Weight

bhama (2006)

sharma (2012)

amra (2014)

van oosten (2014)

foldvary-schaeler (2015)

wong (2015)

Patel (2018)

Tafelmeier (2018)

Overall (I-squared $=50.0 \%, p=0.051)$

NOTE: Weights are from random effects analysis

\begin{tabular}{ll|l}
\hline 1 & & \\
.00042 & 1 & 2407
\end{tabular}

C study

$\begin{array}{clll}131.00(7.13,2406.88) & 10 / 20 & 0 / 65 & 7.41 \\ 4.88(0.60 .39 .91) & 9 / 81 & 1 / 40 & 11.33\end{array}$

$4.88(0.60 .39 .91) \quad 9 / 81 \quad 1 / 40 \quad 11.33$

$3.04(0.26,35.53)-2 / 25 \quad 1 / 36 \quad 9.36$

$\begin{array}{llll}4.61(0.96,22.13) & 8 / 132 & 2 / 145 & 15.23\end{array}$

$0.43(0.09,2.08) \quad 3 / 45 \quad 4 / 28 \quad 15.14$

$2.70(0.68,10.68) \quad 3 / 26 \quad 9 / 195 \quad 16.91$

$2.50(0.73,8.55) \quad 4 / 31 \quad 10 / 179 \quad 18.28$

$10.33(0.41,262.49) \quad 1 / 23 \quad 0 / 77$

$\begin{array}{llll}3.43(1.35,8.71) & 40 / 383 & 27 / 765 & 100.00\end{array}$

\section{cosis}

ID
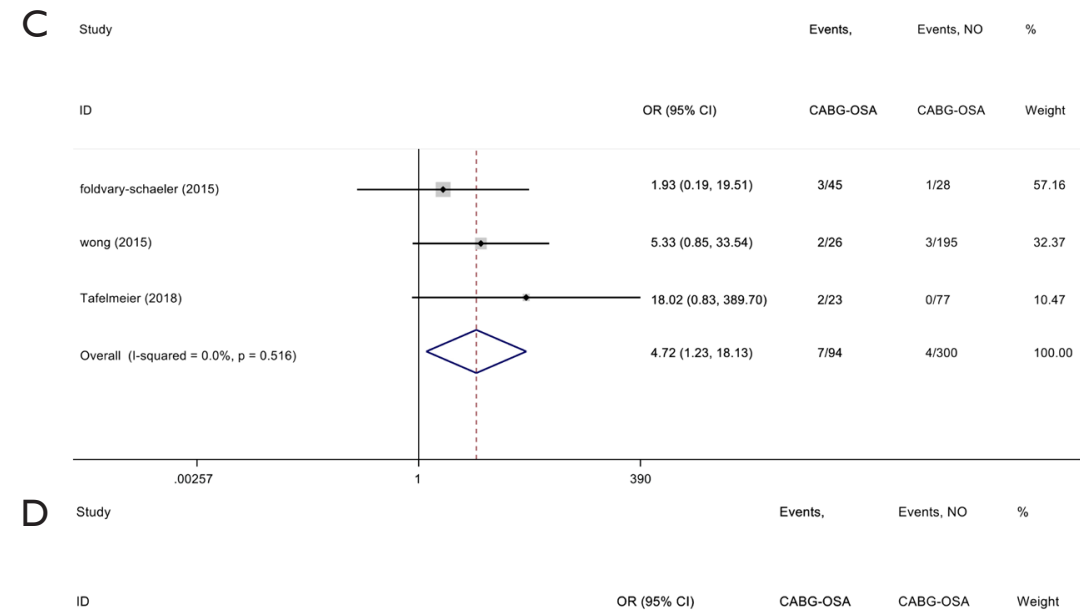

OR $(95 \% \mathrm{Cl}) \quad$ CABG-OSA CABG-OSA Weight

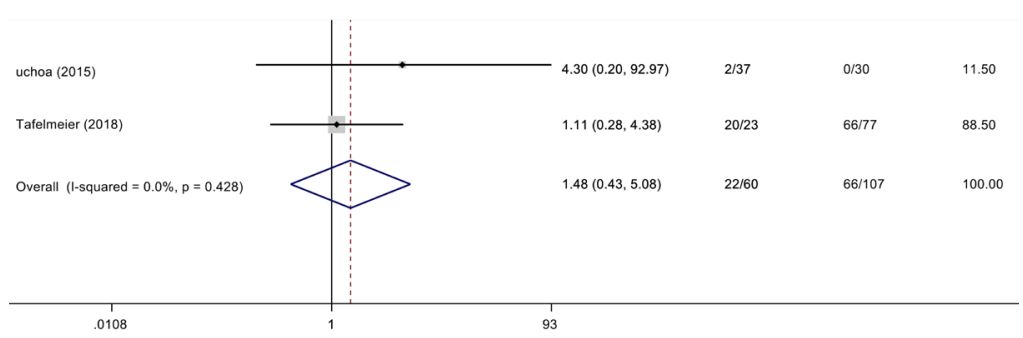

Figure 4 The impact of OSA on postoperative respiratory or airway parameters in CHD patients following elective CABG. Respiratory parameters: (A) Postoperative major pulmonary complications; (B) reintubation; (C) tracheostomy; (D) pulmonary edema. OSA, obstructive sleep apnea; CHD, coronary heart disease; CABG, coronary artery bypass grafting; OR, odds risk; CI, confidence interval. 


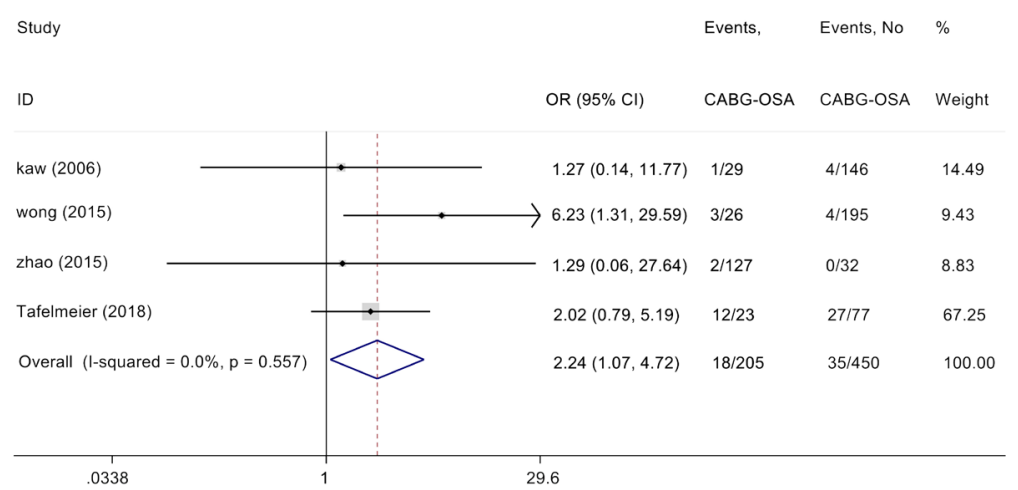

Figure 5 The impact of OSA on postoperative AKI in CHD patients following elective CABG. OSA, obstructive sleep apnea; CHD, coronary heart disease; CABG, coronary artery bypass grafting; AKI, Acute kidney injury; OR, odds risk; CI, confidence interval.

Table 4 Meta-regression for OSA and postoperative composite outcomes

\begin{tabular}{|c|c|c|c|c|c|c|c|c|}
\hline \multirow{2}{*}{ Meta-regression variables } & \multicolumn{2}{|c|}{ MACCEs } & \multicolumn{2}{|c|}{$\begin{array}{l}\text { Major pulmonary } \\
\text { complications }\end{array}$} & \multicolumn{2}{|c|}{ Reintubation } & \multicolumn{2}{|c|}{ AKI } \\
\hline & $\operatorname{Exp}(b)$ & $P$ value & $\operatorname{Exp}(b)$ & $P$ value & $\operatorname{Exp}(b)$ & $P$ value & $\operatorname{Exp}(b)$ & $P$ value \\
\hline Quality of study & 0.517 & 0.479 & 1.047 & 0.126 & 1.004 & 0.379 & 0.725 & 0.884 \\
\hline OSA with CPAP treatment & 0.224 & 0.529 & 1.035 & 0.810 & 0.780 & 0.325 & 1.156 & 0.587 \\
\hline
\end{tabular}

AKI, acute kidney injury; MACCEs, major adverse cardiac and cerebrovascular events; Exp(b) is the value of the risk ratio of continuous variables; PSG, Polysomnography; NE, not enough studies; CPAP, continuous positive airway pressure.

stay (23-25), the current study further demonstrated OSA may contribute to the development of above parameters by causing new or repeated revascularization. The mechanisms underlying OSA-associated adverse cardiac events among CHD patients undergoing CABG may be correlated to the following aspects: endothelial function, arterial stiffening, as well as systemic inflammation. Our previous meta-analysis of 18 studies aimed to investigate the mechanisms underlying OSA-associated cardiovascular events (1). We demonstrated OSA, particularly moderatesevere OSA, was significantly associated with impaired endothelial function (measured by flow mediated dilation and nitroglycerin-induced dilation), increased arterial stiffness (determined by carotid-femoral pulse wave velocity and indicates augmentation index), and elevated serum levels of inflammatory markers (such as high-sensitivity C-reactive protein and C-reactive protein), independent of known confounders (1). The above factors were often used to predict cardiovascular events in general and morbid populations, especially in CHD patients undergoing CABG (26-28). Besides, postoperative troponin T levels, correlated with end-organ damages, suggesting the primary role of an ischemic trigger of end-organ damages (26). Although the recent study demonstrated no differences in the myocardial infarction occurrence in CHD patients undergoing CABG with and without OSA, previous studies indicated the potential role of OSA-associated myocardial ischemia in triggering adverse cardiac events (including POAF) among cardiac surgical patients $(14,29)$. High-quality randomized controlled trials are still needed to confirm. Finally, limited by the original literature, the current meta-analysis confirmed OSA did not affect the incidence of postoperative CVA or TIA. However, in an observational study of 392 men and women with CHD referred for coronary angiography, Valham et al. demonstrated OSA is an independent predictor for stroke among CHD patients (30). 
Possible factors accounting for OSA-associated stroke among CHD patients include apnea-induced nocturnal cerebral ischemia, hypertension, as well as an increased risk of arteriosclerosis (31-33), which are further correlated to stroke. It is worth mentioning that, the independence relationship between sleep-disordered breathing and postoperative heart failure has been confirmed, which contributes to the increased risk of MACCEs (13). Last but not least, previous study demonstrated the rate of D-dimer positivity was higher among cases diagnosed with severe OSA compared to the rest of study population (17.6\%) and indicated that severe-OSA can be a significant risk factor for deep venous thrombosis (34). However, we were limited by the small number of eligible studies and were unable to further examine the correlation between OSA and deep venous thrombosis among CHD patients undergoing CABG. Randomized controlled trials are still needed.

The current study demonstrated there is close relationship between OSA and airway complications, leading to the development of respiratory failure. Reintubation and tracheostomy in the OSA group was increased by $243 \%$ and $372 \%$, respectively, compared with the control group. Both delayed and premature extubation may be correlated to an increased risk of various complications $(35,36)$, and the need for reintubation or tracheostomy was viewed as a potential adverse event after CABG (35-37). Previous studies found that requiring reintubation or tracheostomy was associated with organ dysfunction, especially respiratory failure, secondary to physiologic stress, increased mortality, higher hospital costs and longer length of ICU stay (38-40). Among CHD patients following CABG, OSA was correlated to a longer intubation time $(3,21)$. Guidelines and statements concerning postoperative care in OSA patients undergoing surgery recommend OSA is a relative risk factor for difficult mask ventilation. Plans for difficult airway management should be considered and implemented (41). The current research further confirmed that OSA may contribute to adverse events by increasing the incidence of postoperative reintubation and tracheostomy in CHD patients undergoing CABG. As for major pulmonary complications, previous studies found the patients with OSA undergoing surgery were at a greater risk for increased pulmonary edema, which could further affect the outcomes of the surgery (41-43). However, the impact of OSA on the incidence of major pulmonary complications among CHD patients undergoing CABG still need to be further confirmed by randomized controlled trials.

Currently, no evidence-based studies investigating the effects of OSA on postoperative renal function among CHD patients following CABG. Available reports on the association between OSA and the incidence of AKI after cardiac surgery show conflicting results. In an observational study, Wong et al. found that OSA increased the incidence of postoperative AKI and required renal replacement therapy during hospitalization (16). In contrast, Kaw et al. found that OSA exerted no adverse effects on renal function after cardiac surgery (19). We firstly confirmed OSA significantly increased the AKI incidence (by 124\%) compared with the control group. The mechanism underlying the adverse effects of OSA on renal outcomes among cardiac surgical populations still need to be investigated further. An animal model of sleep apnea demonstrated fibrotic changes and inflammatory in the kidney that were attributed to the impacts of intermittent hypoxia (44). In addition, previous study also reported nocturnal hypoxemia in OSA patients is correlated to accelerated renal dysfunction, possibly because of increased activity of the renal renin-angiotensin system (RAS) as well as glomerular hypertension (45). Moreover, CHD patients following CABG with OSA are also at increased risk of developing preoperative hypertension and diabetes, both of which are closely associated with AKI (46). Moreover, in an observational study, Tafelmeier et al. assessed the incidence of AKI after elective CABG in CHD patients without sleep-disordered breathing (SDB), with central sleep apnea (CSA), or with OSA. They demonstrated OSA was correlated to impaired renal function as observed by significantly higher creatinine values and significantly lower glomerular filtration rates than those of patients in the other two groups (13).

Our results demonstrated that OSA increased medical resource utilization including length of postoperative hospital stay and ICU stay. An increased length of stay (LOS) in ICU for OSA group suggests that CHD patients with OSA may require greater ventilatory support and longer monitoring after CABG, because of OSA-induced postoperative multi-organ dysfunction. Based on this knowledge, peri- and postoperative management of CHD patients with OSA may be optimized to minimize the rate of postoperative cardio-cerebrovascular, respiratory, as well as renal complications, further reducing the consumption of limited medical resources.

Since OSA may contribute to postoperative multi-organ dysfunction among CHD patients undergoing elective CABG, routine sleep apnea screening before CABG need to be conducted for a diagnosis to be firmly established and to determine the type and severity of OSA that will 
dictate treatment alternatives. PSG is the gold standard for confirming the presence of OSA (3). However, PSG is costly and time-consuming (26). This justified the development of ambulatory abbreviated cardiorespiratory recording techniques and establishment of guidelines for the use of these devices (27). In addition, serious complications after CABG have attracted extensive attention increasingly. Previous review study found the rate of CABG-associated in-hospital morbidity and mortality is up to $14.0 \%$ and $2.0 \%$, respectively (47). As for OSA-associated complications, since the current meta-analysis demonstrated that OSA significantly increased the incidence of MACCEs, reintubation, racheostomy and AKI by $97 \%, 243 \%, 372 \%$, and $124 \%$, respectively, among CHD patients undergoing CABG, preventive measurements on OSA in the perioperative phase are needed so as to ultimately improve CHD patient in-hospital outcomes from CABG. It has been confirmed in many relevant literatures perioperative CPAP use in CHD patients with OSA was associated with a decreased rate of cardiac complications after cardiac surgery $(6,14,16)$. Perioperative CPAP use is recommended and may optimize the condition of surgical patients with OSA (6). However, whether perioperative CPAP use would be specifically beneficial to reverse the association of CABGOSA-related respiratory and renal dysfunction, it still needs to be confirmed.

\section{Conclusions}

There are some limitations to the findings of this current research since the absence of randomized controlled trials. Selection bias and treatment bias, inherent to the observational studies, may exist. Moreover, in the current study, heterogeneity was reduced by including only CHD patients alone. Limited by the original literature, we also included CHD patients undergoing CABG + the valvular surgery. Although meta-regression showed the valvular surgery did not affect on our pooled outcomes, high-quality randomized controlled trials focusing on CHD patients undergoing isolated CABG are still needed in the future. In addition, since the sample size of each original studies limitations, currently we cannot further address the effects of mild, moderate as well as severe OSA on multiple organ dysfunction, respectively, in CHD patients undergoing CABG.

In conclusion, the current evidence-based research demonstrated OSA may contribute to postoperative multiorgan dysfunction among CHD patients undergoing elective CABG by increasing the incidence of MACCEs, especially new revascularization (including CABG and PCI), as well as respiratory, and renal complications. Furthermore, our results also demonstrated that OSA increased medical resource utilization including length of postoperative hospital stay and ICU stay. Based on this knowledge, periand postoperative management of CHD patients with OSA may be optimized to minimize the rate of postoperative parameters of multi-organ dysfunction, further reducing the consumption of limited medical resources.

\section{Acknowledgments}

We thank Dr Gui Su for editing and proofreading the manuscript. The statistical analysis was reviewed by Dr Longfei Wang.

Funding: This study was supported by the Beijing Nova of Program.(No.Z201100006820088), the Foundation of Beijing Anzhen Hospital, Capital Medical University (No. 2016Z01), the National Natural Science Foundation of China (Grant No. 81900098), the "Beijing Municipal Administration of Hospitals" Youth Plan (Code: QML20190601), the talents support program of Organization Department of Beijing Municipal Committee (No. 2017000021469G221), the program of the Beijing Municipal Administration of Hospitals (No. XMLX201822).

\section{Footnote}

Reporting Checklist: The authors have completed the PRISMA reporting checklist. Available at http://dx.doi. org/10.21037/jtd-20-2037

Conflicts of Interest: All authors have completed the ICMJE uniform disclosure form (available at http://dx.doi. org/10.21037/jtd-20-2037). The authors have no conflicts of interest to declare.

Ethical Statement: The authors are accountable for all aspects of the work in ensuring that questions related to the accuracy or integrity of any part of the work are appropriately investigated and resolved. The study protocol was reviewed and approved by the ethics committee of all participant institutes in the Beijing An Zhen Hospital Capital Medical University.

Open Access Statement: This is an Open Access article distributed in accordance with the Creative Commons Attribution-NonCommercial-NoDerivs 4.0 International 
License (CC BY-NC-ND 4.0), which permits the noncommercial replication and distribution of the article with the strict proviso that no changes or edits are made and the original work is properly cited (including links to both the formal publication through the relevant DOI and the license). See: https://creativecommons.org/licenses/by-nc-nd/4.0/.

\section{References}

1. Wang J, Yu W, Gao M, et al. Impact of Obstructive Sleep Apnea Syndrome on Endothelial Function, Arterial Stiffening, and Serum Inflammatory Markers: An Updated Meta-analysis and Metaregression of 18 Studies. J Am Heart Assoc 2015;4:e002454.

2. Senaratna CV, Perret JL, Lodge CJ, et al. Prevalence of obstructive sleep apnea in the general population: A systematic review. Sleep Med Rev 2017;34:70-81.

3. Foldvary-Schaefer N, Kaw R, Collop N, et al. Prevalence of Undetected Sleep Apnea in Patients Undergoing Cardiovascular Surgery and Impact on Postoperative Outcomes. J Clin Sleep Med 2015;11:1083-9.

4. Qaddoura A, Kabali C, Drew D, et al. Obstructive Sleep Apnea as a Predictor of Atrial Fibrillation After Coronary Artery Bypass Grafting: A Systematic Review and Metaanalysis. Can J Cardiol 2014;30:1516-22.

5. Chen A, Chen Y. Obstructive Sleep Apnea as a Predictor of Atrial Fibrillation After Coronary Artery Bypass Grafting. Can J Cardiol 2015;31:955.e1.

6. Nagappa M, Ho G, Patra J, et al. Postoperative Outcomes in Obstructive Sleep Apnea Patients Undergoing Cardiac Surgery: A Systematic Review and Meta-analysis of Comparative Studies. Anesth Analg 2017;125:2030-7.

7. Ding N, Ni BQ, Wang H, et al. Obstructive Sleep Apnea: A Risk Factor of Cardiac Valve Replacement Surgery. J Clin Sleep Med 2016;12:1573-5.

8. Feng TR, White RS, Ma X, et al. The effect of obstructive sleep apnea on readmissions and atrial fibrillation after cardiac surgery. J Clin Anesth 2019;56:17-23.

9. Moher D, Liberati A, Tetzlaff J, et al. Preferred reporting items for systematic reviews and meta-analyses: the PRISMA statement. BMJ 2009;339:b2535.

10. Raza S, Sabik JF 3rd, Ainkaran P, et al. Coronary artery bypass grafting in diabetics: A growing health care cost crisis. J Thorac Cardiovasc Surg 2015;150:304-2.e2.

11. Mungan U, Ozeke O, Mavioglu L, et al. The role of the preoperative screening of sleep apnoea by Berlin Questionnaire and Epworth Sleepiness Scale for postoperative atrial fibrillation. Heart Lung Circ
2013;22:38-42.

12. Patel SV, Gill H, Shahi D, et al. High risk for obstructive sleep apnea hypopnea syndrome predicts new onset atrial fibrillation after cardiac surgery: a retrospective analysis. Sleep Breath 2018;22:1117-24.

13. Tafelmeier M, Weizenegger T, Ripfel S, et al. Postoperative complications after elective coronary artery bypass grafting surgery in patients with sleep-disordered breathing. Clin Res Cardiol 2018;107:1148-59.

14. Uchôa CHG, Danzi-Soares NJ, Nunes FS, et al. Impact of OSA on cardiovascular events after coronary artery bypass surgery. Chest 2015;147:1352-60.

15. van Oosten EM, Hamilton A, Petsikas D, et al. Effect of preoperative obstructive sleep apnea on the frequency of atrial fibrillation after coronary artery bypass grafting. Am J Cardiol 2014;113:919-23.

16. Wong JK, Maxwell BG, Kushida CA, et al. Obstructive Sleep Apnea Is an Independent Predictor of Postoperative Atrial Fibrillation in Cardiac Surgery. J Cardiothorac Vasc Anesth 2015;29:1140-7.

17. Zhao LP, Kofidis T, Lim TW, et al. Sleep apnea is associated with new-onset atrial fibrillation after coronary artery bypass grafting. J Crit Care 2015;30:1418.e1-5.

18. Amra B, Niknam N, Sadeghi MM, et al. Obstructive sleep apnea and postoperative complications in patients undergoing coronary artery bypass graft surgery: a need for preventive strategies. Int J Prev Med 2014;5:1446-51.

19. Kaw R, Golish J, Ghamande S, et al. Incremental risk of obstructive sleep apnea on cardiac surgical outcomes. J Cardiovasc Surg (Torino) 2006;47:683-9.

20. Mooe T, Gullsby S, Rabben T, et al. Sleep-disordered breathing: a novel predictor of atrial fibrillation after coronary artery bypass surgery. Coron Artery Dis 1996;7:475-8.

21. Bhama JK, Spagnolo S, Alexander EP, et al. Coronary revascularization in patients with obstructive sleep apnea syndrome. Heart Surg Forum 2006;9:E813-7.

22. Sharma S, Daggubatti R, Tribble RW, et al. Prevalence of Obstructive Sleep Apnea in Patients Undergoing Coronary Artery Bypass Graft Surgery (CABG). A Pilot Study. J Sleep Disord Treat Care 2012;1:2.

23. Massoudy P, Thielmann M, Lehmann N, et al. Impact of prior percutaneous coronary intervention on the outcome of coronary artery bypass surgery: a multicenter analysis. J Thorac Cardiovasc Surg 2009;137:840-5.

24. Thielmann M, Leyh R, Massoudy P, et al. Prognostic significance of multiple previous percutaneous coronary interventions in patients undergoing elective coronary 
artery bypass surgery. Circulation 2006;114:I441-7.

25. Thielmann M, Neuhauser M, Knipp S, et al. Prognostic impact of previous percutaneous coronary intervention in patients with diabetes mellitus and triple-vessel disease undergoing coronary artery bypass surgery. J Thorac Cardiovasc Surg 2007;134:470-6.

26. Wang J, Yu W, Gao M, et al. Continuous positive airway pressure treatment reduces cardiovascular death and nonfatal cardiovascular events in patients with obstructive sleep apnea: A meta-analysis of 11 studies. Int J Cardiol 2015;191:128-31.

27. Vlachantoni IT, Dikaiakou E, Antonopoulos CN, et al. Effects of continuous positive airway pressure (CPAP) treatment for obstructive sleep apnea in arterial stiffness: a meta-analysis. Sleep Med Rev 2013;17:19-28.

28. Drager LF, Togeiro SM, Polotsky VY, et al. Obstructive sleep apnea: a cardiometabolic risk in obesity and the metabolic syndrome. J Am Coll Cardiol 2013;62:569-76.

29. Narducci ML, Pelargonio G, Rio T, et al. Predictors of postoperative atrial fibrillation in patients with coronary artery disease undergoing cardiopulmonary bypass: a possible role for myocardial ischemia and atrial inflammation. J Cardiothorac Vasc Anesth 2014;28:512-9.

30. Valham F, Mooe T, Rabben T, et al. Increased risk of stroke in patients with coronary artery disease and sleep apnea: a 10-year follow-up. Circulation 2008;118:955-60.

31. Peppard PE, Young T, Palta M, et al. Prospective study of the association between sleep-disordered breathing and hypertension. N Engl J Med 2000;342:1378-84.

32. Bålfors EM, Franklin KA. Impairment of cerebral perfusion during obstructive sleep apneas. Am J Respir Crit Care Med 1994;150:1587-91.

33. Shahar E, Whitney CW, Redline S, et al. Sleep-disordered breathing and cardiovascular disease: cross-sectional results of the Sleep Heart Health Study. Am J Respir Crit Care Med 2001;163:19-25.

34. Bahar Y, Annakkaya AN, Sen C, et al. Assessment of the frequency of deep venous thromboembolism in obstructive sleep apnea syndrome. Aging Male 2019. doi: 10.1080/13685538.2019.1654451.

35. Steidl C, Boesel J, Suntrup-Krueger S, et al. Tracheostomy, Extubation, Reintubation: Airway Management Decisions in Intubated Stroke Patients. Cerebrovasc Dis 2017;44:1-9.

36. Peterson GN, Domino KB, Caplan RA, et al. Management of the difficult airway: a closed claims analysis. Anesthesiology 2005;103:33-9.
37. Miltiades AN, Gershengorn HB, Hua M, et al. Cumulative Probability and Time to Reintubation in U.S. ICUs. Crit Care Med 2017;45:835-42.

38. Thille AW, Harrois A, Schortgen F, et al. Outcomes of extubation failure in medical intensive care unit patients. Crit Care Med 2011;39:2612-8.

39. Epstein SK, Ciubotaru RL. Independent effects of etiology of failure and time to reintubation on outcome for patients failing extubation. Am J Respir Crit Care Med 1998;158:489-93.

40. Thille AW, Richard JC, Brochard L. The decision to extubate in the intensive care unit. Am J Respir Crit Care Med 2013;187:1294-302.

41. Ravesloot MJL, de Raaff CAL, van de Beek MJ, et al. Perioperative Care of Patients With Obstructive Sleep Apnea Undergoing Upper Airway Surgery: A Review and Consensus Recommendations. JAMA Otolaryngol Head Neck Surg 2019;145:751-60.

42. Corso R, Russotto V, Gregoretti C, et al. Perioperative management of obstructive sleep apnea: a systematic review. Minerva Anestesiol 2018;84:81-93.

43. Nagappa M, Mokhlesi B, Wong J, et al. The Effects of Continuous Positive Airway Pressure on Postoperative Outcomes in Obstructive Sleep Apnea Patients Undergoing Surgery: A Systematic Review and Metaanalysis. Anesth Analg 2015;120:1013-23.

44. Sun W, Yin X, Wang Y, et al. Intermittent hypoxia-induced renal antioxidants and oxidative damage in male mice: hormetic dose response. Dose Response 2012;11:385-400.

45. Ahmed SB, Ronksley PE, Hemmelgarn BR, et al. Nocturnal hypoxia and loss of kidney function. PLoS One 2011;6:e19029.

46. Marin JM, Agusti A, Villar I, et al. Association between treated and untreated obstructive sleep apnea and risk of hypertension. JAMA 2012;307:2169-76.

47. Lazar HL, Fitzgerald CA, Ahmad T et al. Early discharge after coronary artery bypass graft surgery: Are patients really going home earlier? J Thorac Cardiovasc Surg 2001;121:943-50.

Cite this article as: Wang J, Wang X, Yu W, Zhang K, Wei Y. Obstructive sleep apnea-induced multi-organ dysfunction after elective coronary artery bypass surgery in coronary heart disease patients. J Thorac Dis 2020;12(10):5603-5616. doi: 10.21037/ jtd-20-2037 